\title{
Von einer pragmatischen Theorie der Bedeutung zur Philosophie des Geistes
}

\author{
Michael Esfeld \\ Université de Lausanne, Section de philosophie \\ CH-1015 Lausanne \\ Michael-Andreas.Esfeld@philo.unil.ch
}

(erschienen in André Fuhrmann und Erik J. Olsson (Hgg.):

Pragmatisch denken. Frankfurt (Main): Ontos-Verlag 2004, S. 147-168)

\section{Das Thema des Aufsatzes}

Ein wichtiger Bereich des gegenwärtigen Pragmatismus ist die Theorie der Bedeutung der Aussagen und des begrifflichen Inhalts der Überzeugungen von Personen. Eine pragmatische Theorie der Bedeutung konzipiert die Semantik, die Theorie der Bedeutung sprachlicher Zeichen, auf der Grundlage der Pragmatik, der Theorie des Gebrauchs sprachlicher Zeichen. Sie rekonstruiert die Bedeutung unserer Aussagen und den begrifflichen Inhalt unserer Überzeugungen unter Bezugnahme auf bestimmte soziale Praktiken. Der Pragmatismus in diesem spezifischen Sinne ist der Ausgangspunkt für diesen Aufsatz. Das Ziel ist, eine Antwort auf die Frage zu geben, wie dieser Pragmatismus sich in die gegenwärtige Philosophie des Geistes einfügt.

Im Folgenden spreche ich der Kürze halber nur von »Pragmatismus«, meine damit aber lediglich diese Position, die durch eine Theorie der Bedeutung beziehungsweise des begrifflichen Inhalts auf der Grundlage sozialer Praktiken definiert ist. Ich beschränke mich dabei auf den begrifflichen Inhalt von Überzeugungen. Es geht in diesem Aufsatz also nicht um eine pragmatische Haltung zur Philosophie des Geistes im Sinne einer Haltung, welche die in der Philosophie des Geistes diskutierten Probleme aus pragmatischen Gründen zurückweist. ${ }^{1}$ Es geht vielmehr um die Einordnung einer pragmatischen Theorie der Bedeutung in die gegenwärtige Philosophie des Geistes.

Wenn man eine Theorie entwickelt, welche den begrifflichen Inhalt unserer Überzeugungen von sozialen Praktiken her versteht, dann tut man mehr, als nur einen Beitrag zur Semantik zu leisten. Man stellt dann eine Theorie dessen vor, worin intentionale Zustände bestehen. Intentional sind alle und nur diejenigen Zustände einer Person, die einen begrifflichen, also propositionalen Inhalt haben - wie zum Beispiel zu glauben, dass es regnet, zu hoffen, dass der Zug pünktlich ankommt, zu wollen, dass das Fleisch gut durchgebraten ist und so fort. Die Idee ist, dass eine Person nur dann in intentionalen Zuständen ist, wenn sie an [148] sozialen Praktiken teilnimmt. Eine Theorie intentionaler Zustände ist eine Philosophie des Geistes. Intentionale Zustände sind - neben dem Bewusstsein - der Kernbereich der Philosophie des Geistes. Dementsprechend geht es im Folgenden darum, welche Stelle die Idee, dass die Teilnahme an sozialen Praktiken eine 
notwendige und hinreichende Bedingung für intentionale Zustände ist, in der gegenwärtigen Philosophie des Geistes einnehmen kann.

Dieser Aufsatz stellt ein Problem heraus, statt eine Lösung vorzuschlagen. Der zweite und der dritte Abschnitt erläutern die Motivation und die Grundzüge einer pragmatischen Theorie begrifflichen Inhalts. Der vierte Abschnitt formuliert eine Aufgabe für eine Theorie begrifflichen Inhalts durch soziale Praktiken. Der fünfte Abschnitt zeigt ein Problem auf: Die Behauptung, dass intentionale Zustände real sind, ist nur dann untermauert, wenn gezeigt werden kann, dass intentionale Zustände Auswirkungen auf unser Verhalten haben. Die Weise, wie der begriffliche Inhalt intentionaler Zustände gemäß einer sozialen, pragmatischen Theorie bestimmt wird, scheint jedoch auszuschließen, dass intentionale Zustände für Verhalten ursächlich sein können. Der sechste Abschnitt schließlich zieht auf dieser Grundlage ein kurzes Fazit und weist darauf hin, was man tun können müsste, um dieses Problem zu lösen.

\section{Das Problem des Regelfolgens als Motivation für den Pragmatismus}

Die wichtigste Motivation für eine pragmatische Theorie begrifflichen Inhalts ist das Problem des Regelfolgens. In der heute diskutierten Form geht dieses Problem auf die Philosophischen Untersuchungen von Ludwig Wittgenstein zurück. ${ }^{2}$ Dieses Problem wird von Saul Kripke in dessen Wittgenstein-Interpretation hervorgehoben. ${ }^{3}$ Mit Regelfolgen ist, kurz gefasst, dieses gemeint: Wenn eine Person über einen bestimmten Begriff verfügt, dann hat sie die Fähigkeit, diesen Begriff in unbestimmt vielen neuen Situationen zu verwenden. Wenn eine Person beispielsweise über den Begriff »Baum« verfügt, dann weiß sie, von welchen Dingen es korrekt ist, „Dies ist ein Baum“ zu sagen, und von welchen Dingen es nicht korrekt ist, dieses zu sagen. Wenn diese Person mit einer neuen Situation konfrontiert wird - beispielsweise in eine bisher unerforschte Gegend kommt - dann weiß sie, wie der Begriff »Baum« in dieser neuen Situation korrekt verwendet wird. Das kann man so ausdrücken: Indem eine Person einen Begriff gebraucht, folgt sie einer Regel, die sagt, was korrekt und was inkorrekt in der Verwendung des betreffenden Begriffs ist.

[149] Wittgenstein zeigt gemäß der Interpretation von Kripke: Es gibt an allem Mentalen und allem Naturalen nichts, das über sich selbst hinausweist und einen Begriffsgebrauch für unbestimmt viele neue Situationen etablieren könnte. Gegen die Annahme, dass begrifflicher Inhalt etwas Mentales ist - eine mentale Idee oder Repräsentation -, argumentiert Wittgenstein, dass keine mentale Idee oder Repräsentation über sich selbst hinausweist und festlegen könnte, was die korrekte Verwendungsweise eines bestimmten Begriffs in einer neuen Situation ist. Gegen die Annahme, dass begrifflicher Inhalt durch die Bezugnahme auf Dispositionen zu bestimmtem Verhalten verstanden werden kann, argumentiert Wittgenstein, dass keine Disposition von sich aus in der Lage ist, eine Handlungsweise als korrekt auszuzeichnen. Wittgenstein versucht aufzuweisen: Jeder naturalistische und jeder mentale Kandidat für etwas, in dem der Inhalt unserer Überzeugungen bestehen soll, genügt unendlich vielen logisch möglichen Regeln (begrifflichen Inhalten). Das Problem des Regelfolgens ist daher die Frage, wie wir im Verwenden von Begriffen und damit im Bilden von Überzeugungen bestimmten Regeln folgen können, statt dass unsere Überzeugungen einen beliebigen - und damit gar keinen - Inhalt haben.

2 Wittgenstein (1953), insbesondere $\S \S 138-242$.

3 Kripke (1982) / deutsch Kripke (1987), Kapitel 2. 
Dieses Problem ist die wichtigste Motivation für den Ansatz, den begrifflichen Inhalt unserer Überzeugungen von bestimmten Praktiken aus zu verstehen. Die Pragmatik, um die es dabei geht, ist erstens eine normative Pragmatik: Die Alternative dazu, begrifflichen Inhalt als ein mentales oder ein naturales Faktum anzusehen, besteht darin, begrifflichen Inhalt von bestimmten Normen aus zu konzipieren. Die betreffende Pragmatik ist zweitens eine soziale Pragmatik. Denn einer Person isoliert betrachtet steht kein Kriterium der Unterscheidung zwischen korrektem und inkorrektem Begriffsgebrauch zur Verfügung. ${ }^{4}$ Wenn es etwas Naturales oder Mentales gäbe, das für eine Person isoliert betrachtet ein Kriterium der Unterscheidung zwischen korrektem und inkorrektem Begriffsgebrauch bereitstellen könnte, dann gäbe es ein naturales oder mentales Faktum, das Personen in ihrem Bilden von Überzeugungen vorgegeben ist und an dem der begriffliche Inhalt der Überzeugungen einer Person festgemacht werden könnte. Mit einem Faktum ist hier und im Folgenden immer etwas gemeint, das Personen für ihre intentionalen Einstellungen vorgegeben ist. In einem weiten Sinne des Begriffs »Faktum« können selbstverständlich auch die intentionalen Einstellungen von Personen als etwas Faktisches bezeichnet werden.

\section{Grundzüge einer pragmatischen Theorie begrifflichen Inhalts}

Neben Wittgensteins Philosophischen Untersuchungen ist der Aufsatz „Empiricism and the philosophy of mind“"von Wilfrid Sellars eine wichtige Quelle für [150] eine pragmatische Theorie begrifflichen Inhalts. ${ }^{5}$ Sellars geht nicht auf Wittgenstein und das Problem des Regelfolgens ein. Sein Ausgangspunkt ist die Kritik an einer empiristischen Erkenntnistheorie, gemäß der Sinnesdaten als epistemisches Bindeglied zwischen einen Überzeugungszustand und dessen Referenzobjekt in der Welt treten. Sinnesdaten sind dabei einerseits etwas kausal Gegebenes, andererseits haben sie eine epistemische Funktion - unter anderem die Funktion, das letzte Fundament des Wissens im Sinne der letzten Rechtfertigungsinstanz zu sein. Gegen diese Position, die er als „Mythos des Gegebenen“ abweist, setzt Sellars die folgenden Thesen:

1) Erkenntnis ist begrifflich: Jede Form von Erkenntnis involviert einen begrifflichen Inhalt, der nicht auf Sinnesdaten zurückgeführt werden kann.

2) Begrifflicher Inhalt ist holistisch: Eine Person verfügt nur dann über einen Begriff $F$, wenn sie über eine ganze Reihe weiterer Begriffe verfügt. Denn der begriffliche Inhalt von $F$ ist nicht durch Sinnesdaten, sondern durch seine Beziehungen zu anderen Begriffen bestimmt.

3) Begrifflicher Inhalt ist sozial: Begrifflicher Inhalt ist an Sprache gebunden, und Sprache ist eine soziale Angelegenheit. Kurz, begrifflicher Inhalt wird durch soziale Praktiken bestimmt (und nicht durch Sinnesdaten).

4) Begrifflicher Inhalt ist normativ: Die sozialen Praktiken, die begrifflichen Inhalt bestimmen, sind normativ. In einem intentionalen Zustand zu sein, ist nicht ein naturalistisches Faktum, sondern es heißt, eine bestimmte Position in einem normativen, sozialen System einzunehmen.

Diese Thesen implizieren eine Position, die als Interpretationismus bekannt ist: Von anderen als ein denkendes Wesen interpretiert (behandelt) zu werden und andere als denkende Wesen $\mathrm{zu}$ interpretieren (zu behandeln), ist eine notwendige und hinreichende Bedingung dafür, in

$4 \quad$ Siehe Wittgenstein (1953), § 202.

5 Sellars (1956). Letzte Ausgabe Sellars (1997). Deutsch als Monographie Sellars (1999). 
intentionalen Zuständen zu sein. Denn es ist eine notwendige und hinreichende Bedingung dafür, über einen Begriff $F$ zu verfügen, dass man von anderen so interpretiert (behandelt) wird, dass man über den Begriff $F$ verfügt, und dass man andere so behandelt (interpretiert), dass sie über den Begriff $F$ verfügen. ${ }^{6}$

Der Interpretationismus ist insbesondere mit der Position von Donald Davidson verbunden. Ohne auf Sellars explizit Bezug zu nehmen, vertritt Davidson in seinen Aufsätzen zur Theorie der Interpretation ${ }^{7}$ ebenfalls die genannten vier Thesen. Sein Hauptargument ist ein ähnliches wie das von Wittgenstein. Gemäß Wittgenstein kann eine Person in Isolation betrachtet nicht Regeln folgen, [151] weil ihr keine Unterscheidung zwischen korrektem und inkorrektem Regelfolgen zur Verfügung steht. ${ }^{8}$ Davidson entwickelt sein Argument allerdings unabhängig von Wittgensteins Philosophischen Untersuchungen und der Interpretation von Kripke: Um über einen Begriff $F$ zu verfügen und Überzeugungen zu haben, also in intentionalen Zuständen zu sein, muss man über den Begriff objektiver Wahrheit verfügen. Man muss wissen, dass Überzeugungen einer Unterscheidung zwischen korrekt und inkorrekt (wahr oder falsch) unterworfen sind. Davidson schreibt:

Glauben kann man nur dann etwas, wenn man die Möglichkeit versteht, sich zu irren, und dazu ist nötig, daß man den Gegensatz zwischen Wahrheit und Irrtum - zwischen wahrem Glauben und falschem Glauben - begreift. Dieser Gegensatz kann jedoch, wie ich geltend gemacht habe, nur im Kontext der Interpretation zum Vorschein kommen, der allein uns die Idee einer objektiven öffentlichen Wahrheit aufzwingt. ${ }^{9}$

Das Argument ist also, dass eine Person in Isolation betrachtet nicht über die Unterscheidung zwischen Wahrheit und Irrtum verfügen kann, die eine notwendige Bedingung für intentionale Zustände ist. Dazu ist wechselseitige Interpretation erforderlich.

Anknüpfend an Wittgenstein und Sellars und in Auseinandersetzung unter anderem mit Davidson hat Robert Brandom in seinem Buch Making it explicit die bisher ausführlichste Version einer normativen Pragmatik vorgelegt, die es erlauben soll, begrifflichen Inhalt zu rekonstruieren. ${ }^{10}$ Stellen wir uns eine Situation vor, in der eine Person eine Aussage macht, wie zum Beispiel die Aussage, dass der Kölner Karneval weltbekannt ist. Brandom (1994), Kapitel 1, unterscheidet drei Arten von Normen, unter die eine Person sich stellt, indem sie eine Aussage macht oder eine Überzeugung bildet:

a) Festlegung (commitment): Eine Aussage der Art $p$ zu machen legt eine Person darauf fest, auf Anfrage eine Reihe weiterer Aussagen zu akzeptieren. Wenn man zum Beispiel die Aussage macht, dass der Kölner Karneval weltbekannt ist, ist man auf die Aussage festgelegt, dass in Köln Karneval gefeiert wird.

b) Berechtigung (entitlement): Eine Aussage der Art $p$ zu machen berechtigt eine Person zu einer Reihe weiterer Aussagen. Die Aussage, dass der Kölner Karneval weltbekannt ist, berechtigt eine Person zu der Aussage, dass internationale Medien über den Kölner Karneval berichten werden. [152] Wenn die letztere Aussage angegriffen wird, kann die erstere als Grund angeführt werden.

\footnotetext{
6 Siehe zum Interpretationismus Child (1994), Kapitel 1.

7 in Davidson (1984) / deutsch Davidson (1986).

8 Siehe Wittgenstein (1953), § 202.

9 „Denken und Reden“ in Davidson (1986), S. 246.

10 Brandom (1994) / deutsch Brandom (2000). Eine kompakte Darstellung ist Brandom (2000) / deutsch Brandom (2001).
} 
c) verschlossene Berechtigung (precluded entitlement): Eine Aussage der Art $p$ zu machen verschließt einer Person die Berechtigung zu einer Reihe weiterer Aussagen. Die Aussage, dass der Kölner Karneval weltbekannt ist, verschließt einer Person die Berechtigung zu der Aussage, dass der Kölner Karneval ein bloßes Provinzspektakel ist.

Brandom zufolge ist diese Pragmatik grundlegend: Wir sind denkende Wesen, weil wir uns wechselseitig so behandeln, dass wir auf bestimmte Aussagen festgelegt und zu bestimmten Aussagen berechtigt sind. Wir können in dieser Position zwischen der Rede von Überzeugungen und der Rede von Aussagen wechseln. Etwas ist nur dann eine Überzeugung, wenn es aussagbar ist, weil nur durch die Aussage die Beziehungen der Festlegung, der Berechtigung und der verschlossenen Berechtigung etabliert werden können, in denen begrifflicher Inhalt besteht.

Die genannten pragmatischen Normen bestimmen inferentielle Beziehungen zwischen Aussagen. Die Semantik, zu der diese Pragmatik führt, ist dementsprechend eine inferentielle Semantik. Der begriffliche Inhalt einer Aussage oder einer Überzeugung besteht in inferentiellen Beziehungen zu anderen Aussagen oder Überzeugungen. Die drei genannten pragmatischen Übergänge zwischen Aussagen können wie folgt in inferentielle Beziehungen übersetzt werden:

a) Von Festlegung zu semantischer Implikation: Eine Aussage der Art $p$ impliziert eine Reihe weiterer Aussagen in dem Sinne, dass diese aus jener deduziert werden können.

b) Von Berechtigung zu Unterstützung: Eine Aussage der Art $p$ stützt eine Reihe weiterer Aussagen in dem Sinne, dass eine Induktion zu diesen auf jene gestützt werden kann.

c) Von verschlossener Berechtigung zu Ausschluss: Eine Aussage der Art $p$ schließt eine Reihe weiterer Aussagen aus.

Dieser Übergang von einer normativen Pragmatik zu einer inferentiellen Semantik beinhaltet zwei Thesen:

1) Die inferentiellen Beziehungen, in denen der begriffliche Inhalt einer Aussage oder einer Überzeugung besteht, supervenieren auf normativen Praktiken, sich wechselseitig so zu behandeln, dass man auf etwas festgelegt ist, zu etwas berechtigt ist und dass die Berechtigung zu etwas verschlossen ist.

2) [153] Die Beschreibung des begrifflichen Inhalts im Sinne einer Beschreibung dieser inferentiellen Beziehungen kann im Prinzip auf eine Beschreibung dieser normativen Praktiken reduziert werden.

Allgemein lässt sich die Semantik, auf die diese Position hinausläuft, als eine normative Gebrauchstheorie der Bedeutung fassen: Der begriffliche Inhalt einer Überzeugung eines bestimmten Typs ist durch die Normen der Verwendung von Überzeugungen dieses Typs in einer Gemeinschaft zu einer Zeit festgelegt. Begrifflicher Inhalt lässt sich nicht vollständig explizit machen. Man kann nicht die Festlegungen, Berechtigungen und verschlossenen Berechtigungen, welche den begrifflichen Inhalt einer Überzeugung der Art $P$ ausmachen, abschließend aufzählen. Man kann nur eine Reihe paradigmatischer Beispiele für solche Festlegungen, Berechtigungen und verschlossenen Berechtigungen angeben. Der inferentielle Kontext, in dem begrifflicher Inhalt besteht, ist offen.

Ferner ist begrifflicher Inhalt dieser Semantik zufolge im Fluss: Die Überzeugungen, auf die man durch eine Überzeugung der Art $P$ festgelegt ist, zu denen man berechtigt ist und zu denen eine Berechtigung verschlossen ist, sind nicht ein für alle Mal fixiert. Begrifflicher Inhalt, verstanden als Festlegungen und Berechtigungen, ist wandelbar. Insbesondere 
neuartige Situationen, denen wir begegnen (neue Erfahrungen), können dazu führen, dass neue Festlegungen und Berechtigungen anerkannt werden und manche der bisherigen Festlegungen und Berechtigungen aufgegeben werden. Es gibt demnach keine festen Identitätsbedingungen für begrifflichen Inhalt - weder in der Zeit noch zu einer Zeit.

Diese Position versucht, das Problem des Regelfolgens auf folgende Weise zu vermeiden: Die Praktiken, sich wechselseitig so zu behandeln, dass man auf bestimmte Aussagen festgelegt und zu bestimmten Aussagen berechtigt ist, zeigen, wie einer Person eine Unterscheidung zwischen korrektem und inkorrektem Regelfolgen zur Verfügung stehen kann. Diese Praktiken vermitteln den beteiligten Personen ein praktisches Wissen im Sinne eines Wissens darum, welche Übergänge von einer normativen Einstellung zu anderen normativen Einstellungen angemessen sind, ohne dass diese normativen Einstellungen selbst Gegenstand des Wissens zu sein brauchen. Sie verleihen den Personen auf diese Weise die Fähigkeit, Begriffe in unbestimmt vielen neuen Situationen korrekt zu verwenden.

Gemäß dieser normativen Pragmatik unterliegen intentionale Einstellungen der folgenden Seinsbedingung: Intentionale Einstellungen gibt es nur, insofern Personen sich wechselseitig intentionale Einstellungen zuschreiben, indem sie sich wechselseitig Formen eines normativen Status zuschreiben - auf etwas festgelegt und zu etwas berechtigt zu sein. Nichtsdestoweniger gibt es Wahrheitsbedingungen für die Zuschreibung intentionaler Einstellungen: Einem Wesen intentionale Einstellungen zuzuschreiben ist genau dann wahr, wenn dieses Wesen an einer solchen Praxis des wechselseitigen Zuschreibens intentionaler Einstellungen teilnimmt.

[154] Intentionale Einstellungen unterliegen ferner der folgenden Erkenntnisbedingung: Nur durch Teilnahme an einer Praxis, sich wechselseitig intentionale Einstellungen zuzuschreiben, kann man die intentionalen Einstellungen und deren begrifflichen Inhalt, die in einer solchen Praxis bestimmt sind, erkennen. Das heißt: Die Beschreibung intentionaler Zustände kann nicht auf eine Beschreibung in einem naturalistischen Vokabular reduziert werden. Wenn die Beschreibung intentionaler Zustände auf eine naturalistische Beschreibung reduziert werden könnte, dann bedürfte es keiner pragmatischen Lösung für das Problem des Regelfolgens. Eine naturalistische Lösung im Sinne einer sozialen Variante einer dispositionalen Theorie begrifflichen Inhalts wäre dann zureichend.

\section{Die Rekonstruktion sozialer Praktiken}

Sellars argumentiert dafür, dass begrifflicher Inhalt durch normative, soziale Praktiken bestimmt wird. Er entwickelt jedoch keine Theorie dessen, wie sich der Bereich des Normativen zum Bereich des Nicht-Normativen verhält. Davidsons Ansatz ist eine Theorie radikaler Interpretation. Das ist eine Theorie dessen, wie man aufgrund der Äußerungen eines Sprechers, mit dem man keine Sprache gemeinsam hat, Zugang zu dessen intentionalen Zuständen erlangt. Eine solche Situation setzt offenbar voraus, dass der Sprecher bereits in intentionalen Zuständen ist (Überzeugungen hat). Davidsons Theorie zeigt dann jedoch, dass in intentionalen Zuständen zu sein, wechselseitige Interpretation erfordert: Nur wer an einer sozialen Praxis wechselseitiger Interpretation teilnimmt, hat Überzeugungen. Daraus folgt: Es bedarf einer Konstitutionstheorie dessen, wie man von sozialen Praktiken zu begrifflichem Inhalt gelangt. Wenn diese Konstitutionstheorie nicht naturalistisch und damit nicht reduktionistisch ist, dann muss sie etwas zum Ausgangspunkt nehmen, das einerseits nicht 
naturalistisch ist, andererseits aber auch nicht bereits begrifflichen Inhalt voraussetzt. Ansonsten wäre die Theorie zirkulär.

In Bezug auf eine solche Konstitutionstheorie kann man auf Brandoms Werk Making it explicit verweisen. Wie im letzten Abschnitt skizziert wurde, kann die Beschreibung begrifflichen Inhalts gemäß Brandoms Theorie auf die Beschreibung normativer Praktiken reduziert werden, sich wechselseitig Festlegungen, Berechtigungen und verschlossene Berechtigungen zuzuschreiben.

Die normativen Praktiken, sich wechselseitig so zu behandeln, dass man auf etwas festgelegt und zu etwas berechtigt ist, haben Voraussetzungen im Sinne einer SupervenienzBasis, die naturalistisch beschrieben werden können (auch wenn Brandom selbst diese Voraussetzungen nicht explizit nennt). Wichtig sind insbesondere die folgenden drei Voraussetzungen:

1) Die Personen, die an diesen Praktiken beteiligt sind, haben unabhängig [155] von diesen Praktiken einen kognitiven Zugang zu ihrer Umwelt. Dieser kognitive Zugang besteht zumindest in zuverlässigen und unterscheidenden Reaktionsmechanismen, die durch mentale Repräsentationen gesteuert sind. Er umfasst keinen begrifflichen Inhalt. Die normativen Praktiken, welche den begrifflichen Inhalt von Überzeugungen bestimmen, setzen somit nicht nur die Existenz einer physikalischen Welt voraus, sondern auch einen kognitiven, nicht-begrifflichen Zugang zu dieser Welt. Diese Voraussetzung ist keine Grundlage für einen Einwand gegen eine pragmatische Theorie begrifflichen Inhalts. Das Problem des Regelfolgens kann als Argument dafür angesehen werden, dass eine mentalistische Erkenntnistheorie scheitert. Für ein Subjekt in Isolation von anderen Subjekten und in Isolation von einer physikalischen Welt betrachtet gibt es nichts, das diesem Subjekt einen begrifflichen Inhalt für Überzeugungen geben könnte. Eine soziale und eine physikalische Umwelt mitsamt einem kognitiven Zugang zu dieser Umwelt sind eine notwendige Bedingung für Regelfolgen.

2) Die Personen, die an diesen Praktiken beteiligt sind, haben unabhängig von diesen Praktiken eine Disposition, das eigene Verhalten zumindest partiell mit dem Verhalten ihrer Mitmenschen zu koordinieren. Das ist eine Disposition zweiter Ordnung - eine Disposition, einiges von den eigenen Dispositionen und dem eigenen Verhalten infolge des Verhaltens der Mitmenschen zu ändern. Diese Veränderung braucht kein bewusster Vorgang zu sein. Eine solche Disposition zu haben und auszuüben ist eine notwendige und hinreichende Bedingung dafür, dass Verhalten soziales Verhalten ist. Soziales Verhalten kann dieser Einteilung zufolge naturalistisch verstanden werden.

3) Aufgrund der Disposition zu zumindest partieller Koordination reagieren die Personen, die an diesen Praktiken beteiligt sind, unabhängig von diesen Praktiken auf ihr Verhalten so, dass sie wechselseitig Sanktionen im Sinne von Bestärkungen und Zurückhaltungen anwenden. Sie bestärken das Verhalten in anderen, das ihrem eigenen Verhalten gleicht, und sie halten das Verhalten in anderen zurück, das ihrem eigenen Verhalten nicht gleicht. Sanktionen unterstützen auf diese Weise einen Prozess, der zu Konvergenz im Verhalten führt.

Sanktionen im Sinne von Punkt 3 sind ausschließlich Bestärkungen und Zurückhaltungen von Verhalten, die vollständig in naturalistischem Vokabular beschrieben werden können. Sanktionen in diesem Sinne sind das charakteristische Merkmal sozialen Verhaltens: Etwas 
ist nur dann soziales Verhalten, wenn die [156] beteiligten Wesen wechselseitig durch Bestärkungen und Zurückhaltungen auf ihr Verhalten reagieren.

Soziale Praktiken liegen genau dann vor, wenn die Sanktionen normativ sind: Es sind nicht bloße Bestärkungen und Zurückhaltungen von bestimmtem Verhalten, sondern normative Einstellungen der Beteiligten, in denen die Beteiligten ihr Verhalten wechselseitig als korrekt oder inkorrekt beurteilen und sich damit Formen eines normativen Status zusprechen - in Form von Festlegungen, Berechtigungen und verschlossenen Berechtigungen. Verhalten, das für korrekt gehalten wird, wird bestärkt; Verhalten, das für inkorrekt gehalten wird, wird zurückgehalten. Der Maßstab, anhand dessen ein Wesen das Verhalten von anderen für korrekt oder inkorrekt hält, kann zunächst die Gleichheit oder Ungleichheit mit dem eigenen Verhalten sein. In einem weiteren Schritt können Sanktionen im normativen Sinne selbst einer Beurteilung unterworfen werden, ob diese Sanktionen korrekt oder inkorrekt sind. ${ }^{11}$

Der Unterschied zwischen sozialem Verhalten und sozialen Praktiken beruht demnach auf normativen Einstellungen: Soziale Praktiken liegen genau dann vor, wenn die beteiligten Personen zueinander normative Einstellungen einnehmen. Aufgrund dieser Einstellungen sprechen sich Personen wechselseitig normative Status zu (Festlegungen, Berechtigungen und verschlossene Berechtigungen). Mit diesen Status ist dann ein begrifflicher Inhalt für intentionale Zustände etabliert. Um diesem Ansatz Plausibilität zu verleihen, müssen zwei Aufgaben erfüllt werden:

- Einerseits muss man das, was mit normativen Einstellungen gemeint ist, so erläutern können, dass normative Einstellungen nicht begrifflichen Inhalt voraussetzen. Ansonsten wäre die Theorie zirkulär. Man kann zumindest Folgendes sagen: Normative Einstellungen sind Einstellungen, etwas für korrekt oder inkorrekt zu halten. Solche Einstellungen setzen noch nicht voraus, ein Kriterium der Unterscheidung zwischen korrekt und inkorrekt zur Verfügung zu haben. Ein solches Kriterium geben erst die genannten sozialen Praktiken. Ein solches Kriterium ist aber gemäß dem Argument von Wittgenstein und Davidson die Voraussetzung dafür, über Begriffe zu verfügen - und somit die Voraussetzung dafür, Überzeugungen (intentionale Zustände) zu haben. Insofern kann es normative Einstellungen geben, ohne dass begrifflicher Inhalt bestimmt ist.

- Andererseits muss für die Beschreibungen normativer Einstellungen ein AntiReduktionsargument gelten, wenn die pragmatische Lösung des Problems des Regelfolgens nicht eine - raffinierte - Variante einer [157] naturalistischen Lösung unter Bezugnahme auf bestimmte Dispositionen zu sozialem Verhalten sein soll: Obwohl normative Einstellungen noch nicht begrifflich sind, gibt es keine Beschreibung von Verhaltensdispositionen, auf welche die Beschreibung normativer Einstellungen reduziert werden kann. Das heißt: Normativität muss unabhängig von begrifflichem Inhalt als AntiReduktionsargument ausgewiesen werden können, um diese Position plausibel zu machen.

Wenn diese beiden Aufgaben erfüllt werden können, folgt: Normative Einstellungen sind selbstverständlich im Laufe der Evolution aus Nicht-Normativem entstanden. Über die Angabe einer Supervenienz-Basis hinaus gibt es jedoch keine Möglichkeit, normative, soziale

11 Vergleiche in diesem Zusammenhang Pettit (1993), S. 76-108, Haugeland (1998), S. 147-150, und Esfeld (2002), Kapitel 3.2. 
Praktiken von nicht-normativem, sozialem Verhalten - und weiter gefasst, von einer naturalistischen Beschreibung dessen, was es in der Welt gibt - aus zu rekonstruieren.

\section{Das Problem der mentalen Verursachung}

Ein Haupteinwand gegen den Interpretationismus lautet, dass diese Position nicht die Realität intentionaler Zustände ausweisen kann. Die normativen, sozialen Praktiken, in denen wir uns wechselseitig intentionale Zustände zuschreiben, sind ein Spiel, in dem wir uns so interpretieren, als ob wir in intentionalen Zuständen wären. Diese Praktiken konstituieren aber nicht eine Realität intentionaler Zustände. Vor allem der Interpretationismus von Daniel Dennett wird häufig als eine solche anti-realistische Position in Bezug auf intentionale Zustände ausgelegt. ${ }^{12}$ Insbesondere dann, wenn eine normative, soziale Theorie begrifflichen Inhalts zirkulär ist - also begrifflichen Inhalt nicht auf der Grundlage von etwas rekonstruieren kann, das nicht bereits begrifflichen Inhalt voraussetzt - provoziert sie diesen Einwand. Diesem Einwand könnte man dann nur durch eine idealistische Metaphysik ausweichen - das heißt, eine Metaphysik, gemäß der die Welt selbst begrifflich ist oder von begrifflichem Inhalt abhängig ist. ${ }^{13}$ Für eine solche Metaphysik spricht jedoch nichts. Deshalb ist es so wichtig, dass eine soziale, pragmatische Theorie begrifflichen Inhalts den Zirkularitätseinwand vermeiden kann im Sinne der beiden Aufgaben, die im letzten Abschnitt benannt wurden. Denn das Hauptargument für eine naturalistische Lösung für das Problem des Regelfolgens ist, dass nur eine solche Position die Realität intentionaler Zustände ausweisen kann.

[158] Nehmen wir an, die beiden Aufgaben, die im vorigen Abschnitt benannt wurden, könnten erfüllt werden. Selbst dann wäre das größte Problem noch nicht gelöst, das sich für das Projekt stellt, intentionale Zustände von sozialen Praktiken aus zu verstehen: Wie können intentionale Zustände, so konzipiert, Auswirkungen auf unser Verhalten haben? Die Möglichkeit, einen Epiphänomenalismus in Bezug auf intentionale Zustände zu vertreten, besteht für diese Position nicht. Denn der begriffliche Inhalt intentionaler Zustände ist durch Beziehungen des Übergangs zu anderen intentionalen Zuständen einschließlich Handlungen definiert, und dieser Übergang ist kausal. Um die Realität intentionaler Zustände auszuweisen, muss diese Position also darlegen können, wie intentionale Zustände - auch physikalische Wirkungen haben können.

Damit stellt sich für diese Position das bekannte Problem der mentalen Verursachung. Dieses Problem wird im allgemeinen anhand von vier Prinzipien eingeführt. Jedes dieser Prinzipien ist für sich genommen plausibel. Sie können aber nicht alle vier zusammen in der vorliegenden Form wahr sein:

1) Verschiedenheit: Intentionale Zustände sind von physikalischen Zuständen verschieden. Um diese Verschiedenheit zu begründen, reicht im Rahmen der hier diskutierten Position der Verweis darauf hin, dass der begriffliche Inhalt intentionaler Zustände von irreduzibel normativen, sozialen Praktiken abhängig ist.

2) mentale Verursachung: Intentionale Zustände verursachen physikalische Zustände.

12 Grundlage dafür ist insbesondere Dennett (1987). Dennett hat seine Position inzwischen abgeschwächt, um der anti-realistischen Interpretation entgegenzutreten. Siehe insbesondere Dennett (1991). 
3) Vollständigkeit physikalischer Ursachen: Für alle physikalischen Zustände gilt: Insofern ein physikalischer Zustand eine Ursache hat, hat er eine vollständige physikalische Ursache.

4) keine systematische Überdetermination: Intentionale Zustände verursachen physikalische Zustände nicht dadurch, dass die betreffenden physikalischen Zustände systematisch überdeterminiert sind durch vollständige physikalische Ursachen und zusätzliche mentale Ursachen.

Das dritte Prinzip basiert auf den Naturwissenschaften: Physikalische Kausalität ist an Naturgesetze gebunden, und die Naturgesetze enthalten keine Lücken, die mentale Ursachen zulassen - oder gar verlangen.

Wenn man (3) aufgibt, steht man vor einem Dilemma: Das eine Horn des Dilemmas ist die Konsequenz, dass einige Gesetzesaussagen naturwissenschaftlicher Theorien falsch sind aufgrund von mentaler Verursachung. Wenn mentale Ursachen verschieden von physikalischen Ursachen sind und physikalische Wirkungen haben, ohne dass diese Wirkungen überdeterminiert sind, dann sind [159] einige naturwissenschaftliche Gesetzesaussagen falsch, weil sie nicht die korrekten Wahrscheinlichkeiten für das Eintreten bestimmter physikalischer Zustände angeben. Immer wenn eine Person beispielsweise die Absicht hat, ihren rechten Arm zu heben, dann wird durch diese Absicht das Eintreten bestimmter neurophysiologischer Zustände, die für das Hochgehen des Armes erforderlich sind, wahrscheinlicher als es ohne diese Absicht wäre. Eine entsprechende Überlegung gilt auch, wenn man bis auf die Ebene der Mikrophysik und der Gesetzesaussagen der Quantenmechanik hinuntergeht. ${ }^{14}$ Dieser Konsequenz kann man in dem Rahmen, der durch (1), (2) und (4) gegeben ist, nur dadurch entgehen, dass man sagt, die naturwissenschaftlichen Gesetze gelten für einige physikalische Zustände nicht: Das Gehirn - oder ein bestimmter Bereich des Gehirns - ist kein abgeschlossenes physikalisches System und kein Teil eines abgeschlossenen physikalischen Systems, weil es in Interaktion mit einem nichtphysikalischen System, dem Geist, steht. Deshalb fallen zumindest einige Gehirnzustände aus dem Bereich dessen heraus, auf den die naturwissenschaftlichen Gesetzesaussagen anwendbar sind. ${ }^{15}$ Das ist das andere Horn des Dilemmas. Aufgrund dieser implausiblen Konsequenzen wird ein interaktionistischer Dualismus in der gegenwärtigen Diskussion kaum noch vertreten.

Unter der Voraussetzung, (1) und (2) unangetastet zu lassen, sind in den letzten Jahren statt der Aufgabe von (3) eher Positionen entwickelt worden, welche eine Art systematischer Überdetermination der physikalischen Zustände, die auch mentale Ursachen haben, plausibel $\mathrm{zu}$ machen versuchen und somit (4) aufgeben. ${ }^{16}$ Es ist jedoch mehr als fraglich, ob man mit der Annahme von Überdetermination zu einer überzeugenden Lösung für das Problem der mentalen Verursachung gelangen kann.

Wenn man an (3) und (4) festhält und (2) anerkennt, dann muss man (1) modifizieren. Man ist in diesem Fall auf die These festgelegt, dass die mentalen Ursachen physikalischer Zustände mit physikalischen Ursachen identisch sind. Nur dann, wenn die mentalen Ursachen mit physikalischen Ursachen identisch sind, können diese physikalische Wirkungen haben,

14 Siehe dazu Esfeld (2000).

15 Vergleiche Averill \& Keating (1981).

16 Für verschiedene Ansätze in diesem Sinne siehe vor allem Yablo (1992), Loewer (2001) und Bennett (2003). 
gegeben (3) und (4). Kausalität wird im allgemeinen als eine Beziehung zwischen einzelnen Vorkommnissen (,tokens“ im Unterschied zu „types“) angesehen: Ein Vorkommnis eines mentalen Zustands einer bestimmten Art verursacht ein Vorkommnis eines physikalischen Zustands einer bestimmten Art. Gegeben (2), (3) und (4) folgt dann, dass jedes Vorkommnis eines mentalen Zustands, der physikalische Wirkungen hat, identisch mit einem Vorkommnis eines physikalischen Zustands ist.

[160] Diese Konsequenz entspricht der Position von Davidson. Davidson ist der einzige der vier Autoren, die in den vorigen Abschnitten in Bezug auf eine pragmatische Theorie begrifflichen Inhalts erwähnt wurden, welcher eine Theorie der mentalen Verursachung entwickelt. Davidson spricht von Ereignissen statt von Zuständen. Mentale Verursachung dient ihm als Argument dafür, zu vertreten, dass jedes mentale Ereignis mit einem physikalischen Ereignis identisch ist. Nach Davidson ist Kausalität eine Beziehung zwischen Ereignissen unabhängig davon, wie diese Ereignisse beschrieben werden. Alle Ereignisse erfüllen eine physikalische Beschreibung (das heißt, machen eine physikalische Beschreibung wahr). Einige Ereignisse erfüllen auch eine mentale Beschreibung. ${ }^{17}$

Dennoch wird gegen Davidson der Einwand des Epiphänomenalismus erhoben. ${ }^{18}$ Kausalität ist nach Davidson an strikte (das heißt, ausnahmslos geltende) Gesetze gebunden, und solche Gesetze gibt es nur in fundamentalen Theorien der Physik. Der Einwand ist daher, dass Davidson nicht zeigen kann, wie Ereignisse, insofern sie mental sind, für etwas ursächlich sein können. Um ein Beispiel von Dretske aufzunehmen, ${ }^{19}$ die Stimme der Sängerin bringt ein Glas zum Zerspringen, aber es ist die Tonhöhe und die Tonintensität, und nicht der begriffliche Inhalt des Gesangs, aufgrund dessen das Glas zerspringt. Für diesen Effekt ist der begriffliche Inhalt des Gesangs epiphänomenal. Der Einwand gegen Davidson lautet, dass seine Theorie zur Folge hat, dass sich begrifflicher Inhalt immer wie in diesem Beispiel verhält.

Um diesen Einwand auszuräumen, muss man zeigen, wie dasjenige, was eine physikalische Beschreibung wahr macht, als dasselbe auch eine mentale Beschreibung wahr macht. Mit anderen Worten: Man braucht eine feingliedrigere Konzeption von Ereignissen oder Zuständen als diejenige von Davidson. Eine solche Konzeption erreicht man, wenn man einen Zustand als die Instantiation einer Eigenschaft betrachtet. Mentale Kausalität erfordert dann, gegeben (3) und (4), dass jede Instantiation einer mentalen Eigenschaft, die ursächlich für die Instantiation einer physikalischen Eigenschaft ist, mit etwas Physikalischem identisch ist. Kurz, die Identität der Vorkommnisse muss sich auf die Vorkommnisse von Eigenschaften beziehen.

Der Funktionalismus scheint die naheliegende - und die einzige - Möglichkeit zu sein, eine solche Identität der Vorkommnisse auszuführen, ohne auf einen reduktiven Physikalismus festgelegt zu sein. Gemäß dem Standard-Funktionalismus kausaler Rollen wird ein mentaler Zustand vom Typ $M$ durch seine typischen Ursachen und Wirkungen definiert. Es wird also durch Definition [161] gefordert, dass jeder mentale Zustand kausal wirksam ist. Die betreffende Rolle ist physikalisch realisiert. Das heißt, es gibt Anordnungen physikalischer Zustände, letztlich mikrophysikalischer Zustände, welche die typischen Ursachen und

17 Siehe Davidson (1970) / deutsch in Davidson (1985), Kapitel 11.

18 Siehe insbesondere die Diskussion zwischen Davidson (1993), Kim (1993), McLaughlin (1993) und Sosa (1993).

19 Dretske (1989), S. 1-2. 
Wirkungen exemplifizieren, die einen mentalen Zustand des Typs $M$ definieren. Deshalb sind diese Anordnungen physikalischer Zustände ein Fall von $M$. Physikalische Realisation wird in der Regel so aufgefasst, dass jedes Vorkommnis von $M$ mit einem Arrangement von Vorkommnissen physikalischer Zustände identisch ist oder durch ein Arrangement von Vorkommnissen physikalischer Zustände konstituiert wird. ${ }^{20}$ Daraus folgt, dass es für jeden einzelnen Fall von $M$ eine physikalische Beschreibung gibt (auch wenn es - vielleicht prinzipielle - epistemische Hindernisse dafür geben mag, dass wir diese Beschreibung kennen). Die betreffende Beschreibung impliziert ein Gesetz: Jeder Fall eines solchen Arrangements ist ein Fall von $M$ - und zwar mit metaphysischer Notwendigkeit, weil jeder solche Fall die Definition von $M$ erfüllt. Diese Beschreibung enthält ferner eine Erklärung: Es gibt Vorkommnisse von $M$ in der Welt, weil es Arrangements physikalischer Zustände gibt, welche die für $M$ typischen Ursachen und Wirkungen aufweisen.

Dennoch ist der Standard-Funktionalismus nicht auf einen reduktiven Physikalismus festgelegt: $M$ kann vielfältig realisiert sein. Alles, was die Arrangements physikalischer Zustände, welche $M$ realisieren, gemeinsam haben müssen, ist, bestimmte Ursachen und Wirkungen aufzuweisen. Diese Arrangements können aber physikalisch ganz verschiedenartig zusammengesetzt sein. Intentionale Zustände desselben Typs können sowohl in menschlichen Gehirnen als auch in Super-Computern realisiert sein. Infolgedessen kann die Beschreibung von $M$ als Typ nicht auf die Beschreibung eines physikalischen Typs reduziert werden. Der Typ $M$ ist nicht mit einem physikalischen Typ identisch. Insofern respektiert der Standard-Funktionalismus das Prinzip der Verschiedenheit von mentalen und physikalischen Zuständen (1) - wenn auch nicht auf der Ebene der einzelnen Vorkommnisse (tokens), so doch auf der Ebene der Typen.

Der Standard-Funktionalismus definiert mentale Zustände durch eine kausale Rolle, die auf die jeweilige Person beschränkt ist. Dieses Definitionsverfahren ist mit einer sozialen, normativen Theorie begrifflichen Inhalts nicht vereinbar. Dennoch kann diese Theorie auch als eine Art Funktionalismus aufgefasst werden, und zwar als ein sozialer Funktionalismus: Der begriffliche Inhalt eines intentionalen Zustands vom Typ $M$ ist dessen soziale Rolle, definiert im oben genannten Sinne durch Beziehungen der Festlegung, der Berechtigung und der verschlossenen Berechtigung. Mit anderen Worten, der begriffliche Inhalt eines intentionalen Zustands ist seine Position innerhalb einer Lebensform im Sinne Wittgensteins.

[162] Lässt sich die Lösung für das Problem der mentalen Verursachung, welche der Standard-Funktionalismus kausaler Rollen bietet, wenn er im Sinne einer Identität der Vorkommnisse ausgeführt wird, ${ }^{21}$ auf den sozialen Funktionalismus übertragen? Auch und gerade im Rahmen des sozialen Funktionalismus kann man vertreten, dass intentionale Zustände physikalisch realisiert sind. Man kann das Problem des Regelfolgens als ein Argument dagegen einsetzen, etwas Mentales anzuerkennen, das nicht physikalisch realisiert ist - auf der Grundlage dessen, dass dieses Problem gegen eine mentalistische Semantik spricht. Fraglich ist jedoch, ob man die Weise, wie der Standard-Funktionalismus die

20 Siehe zum Beispiel Shoemaker (1981) und Poland (1994), S. 16-18.

21 Vor allem Kim (1998) kritisiert den Standard-Funktionalismus, der ein nicht-reduktiver Physikalismus ist, als inkohärent. Für den Zweck dieses Aufsatzes nehme ich jedoch ohne weitere Begründung an, dass ein nicht-reduktiver Physikalismus trotz der Einwände von Kim eine kohärente Position ist. Siehe gegen Kim insbesondere Antony \& Levine (1997). 
physikalische Realisation als Identität der Vorkommnisse ausführt, um das Problem der mentalen Verursachung zu lösen, auf den sozialen Funktionalismus übertragen kann.

Der These der Identität der Vorkommnisse steht zunächst der Holismus intentionaler Zustände entgegen: Ein intentionaler Zustands des Typs $M$ ist definiert durch normative Beziehungen zu intentionalen Zuständen anderer Typen und Konsequenzen für das Verhalten. Letztere Konsequenzen sind zwar physikalisch beschreibbar, reichen aber in keinem Falle aus, um einen intentionalen Zustand zu definieren (das ist die Lehre aus dem Scheitern des Behaviourismus, die der Funktionalismus zieht). Ein intentionaler Zustand eines Typs ist im wesentlichen durch seine Beziehungen zu intentionalen Zuständen anderer Typen bestimmt. Die Frage ist daher, ob es genau ein Arrangement von Vorkommnissen physikalischer Zustände gibt, mit dem ein gegebenes Vorkommnis eines intentionalen Zustandes - im Unterschied zu Vorkommnissen anderer intentionaler Zustände - identisch ist. Nehmen wir an, eine Person ist im intentionalen Zustand $p$, ein Zustand vom Typ $P$ ist bestimmt durch Beziehungen zu Zuständen der Art $Q, R$ und $S$, und die betreffende Person ist zugleich auch in den intentionalen Zuständen $q, r$ und $s$. Gibt es ein Arrangement von Vorkommnissen physikalischer Zustände, mit denen $p$ im Unterschied zu $q, r$ und $s$ identisch ist?

Man kann diese Frage so zu beantworten versuchen, dass man sagt, dass das Netzwerk der Vorkommnisse der intentionalen Zustände $p, q, r$ und $s$ mit einem Netzwerk von Vorkommnissen physikalischer Zustände identisch ist. Das würde darauf hinauslaufen, den gesamten intentionalen Zustand einer Person zu einer Zeit mit dem gesamten Gehirnzustand der Person zu dieser Zeit zu identifizieren. Diese Antwort ist für das Problem der mentalen Verursachung jedoch unzureichend: Es ist nicht der Fall, dass $p, q, r$ und $s$ nur zusammen physikalische Wirkungen haben. Vielmehr hat jedes dieser Vorkommnisse spezifische physikalische Wirkungen - oder leistet zumindest einen spezifischen Beitrag zu einer physikalischen [163] Gesamtwirkung. Gemäß dem oben Ausgeführten ist die kausale Wirksamkeit von jedem dieser Vorkommnisse nur dann sichergestellt, wenn jedes dieser mentalen Vorkommnisse mit einem Arrangement von physikalischen Vorkommnissen identisch ist.

Um eine Antwort auf das Problem der mentalen Verursachung in dem genannten Rahmen zu geben, muss man daher eine Theorie der Identität von Vorkommnissen - im Unterschied zu einer Theorie der Identität von Typen - entwickeln können, die folgendes zeigt: Jedes Vorkommnis eines intentionalen Zustands (jedes Vorkommnis einer mentalen Eigenschaft) ist mit einem Arrangement von Vorkommnissen physikalischer Zustände identisch, obwohl das betreffende Vorkommnis eines intentionalen Zustandes das, was es ist, aufgrund seiner Beziehungen zu anderen Vorkommnissen intentionaler Zustände ist. Es ist hingegen zulässig, dass das betreffende Arrangement physikalischer Zustände das, was es ist, unabhängig von Vorkommnissen außerhalb dieses Arrangements ist. $\mathrm{Zu}$ beachten ist, dass das HolismusProblem sich auch schon für den Standard-Funktionalismus stellt. Denn auch gemäß dem Standard-Funktionalismus wird ein intentionaler Zustand eines Typs im wesentlichen durch seine Beziehungen zu intentionalen Zuständen anderer Typen definiert.

Für den sozialen Funktionalismus ergibt sich ein weiteres Problem, das als noch gravierender gilt: Gemäß dem sozialen Funktionalismus besteht der begriffliche Inhalt eines intentionalen Zustands in Beziehungen zu anderen intentionalen Zuständen, die durch soziale Praktiken bestimmt werden. Der begriffliche Inhalt eines intentionalen Zustands ist also von externen Faktoren abhängig. Die kausalen Wirkungen, die ein physikalischer Zustand 
entfalten kann, sind hingegen in der Regel nicht von externen Faktoren abhängig. Gehirnzustände können bestimmte körperliche Bewegungen verursachen unabhängig davon, wie die soziale Umwelt beschaffen ist.

Ein Externalismus in Bezug auf begrifflichen Inhalt ist weitgehend anerkannt. Die gängige Strategie, um dem Externalismus-Problem für mentale Kausalität zu begegnen, besteht darin, zwischen einem engen und einem weiten begrifflichen Inhalt zu unterscheiden. Der enge begriffliche Inhalt ist nur von internen Faktoren abhängig. Dieser ist derjenige begriffliche Inhalt, welcher für das Verhalten der Person ursächlich ist. Im Rahmen des sozialen Funktionalismus ist diese Unterscheidung zwischen engem und weitem begrifflichen Inhalt jedoch nicht möglich: Jede Art von begrifflichem Inhalt ist weit, da jede Art von begrifflichem Inhalt durch soziale Praktiken bestimmt ist. 22

Nichtsdestoweniger kann man sagen, dass der intentionale Zustand ein Zustand der betreffenden Person ist. Die Person ist in einem Zustand, der mit anderen Zuständen der Person verbunden ist. Wenn diese Verbindungen bestimmten sozialen Normen genügen, dann haben diese Zustände einen [164] bestimmten begrifflichen Inhalt. In diesem Sinne sind intentionale Zustände auch gemäß einem sozialen Funktionalismus im Kopf beziehungsweise in der Person. Um das Problem der mentalen Verursachung in dem genannten Rahmen zu lösen, könnte man auf dieser Basis versuchen, trotz des sozialen Externalismus für eine Identität zwischen Vorkommnissen von intentionalen Zuständen und Vorkommnissen von Gehirnzuständen zu argumentieren.

Wiederum gilt, dass das Externalismus-Problem nicht auf eine soziale, pragmatische Theorie begrifflichen Inhalts beschränkt ist. Es trifft auch die derzeit populärste Form einer Naturalisierung der Semantik, die Teleo-Semantik. Ruth Garrett Millikan, eine Hauptvertreterin des Programms, Typen begrifflichen Inhalts mit Typen biologischer Funktionen zu identifizieren und so begrifflichen Inhalt auf biologische Funktionen zu reduzieren, schreibt:

... the semantic category of a thought is determined relative to its biological functions, which depend in turn upon its history, upon its place relative to certain prior events. But having a certain history is not, of course, an attribute that has 'causal powers'. Hence reasons cannot be, as such, causes. More generally, that a thing has a teleo-function is a causally impotent fact about it. 23

Das heißt: Der Epiphänomenalismus-Einwand kann gegen die biologische Reduktion der Semantik in gleicher Weise wie gegen Davidson erhoben werden.

\section{6. $\quad$ Der Stand der Kunst: das Problem der Erklärungslücke}

In der Philosophie des Bewusstseins ist in Bezug auf die Zustände phänomenalen Erlebens die sogenannten Qualia-Zustände, wie zum Beispiel Schmerzen zu empfinden, die Farbe rot zu sehen etc. - ein Problem der Erklärungslücke weitgehend anerkannt. Es ist zumindest nicht offensichtlich, wie der Standard-Funktionalismus zusammen mit der These der physikalischen Realisierung mentaler Zustände den Erlebnischarakter dieser Zustände erfassen kann. Insofern besteht eine Erklärungslücke zwischen der funktionalen Definition von Bewusstseinszuständen und deren Erlebnischarakter. ${ }^{24}$ Umstritten ist, ob diese

22 Vergleiche Esfeld (2003).

23 Millikan (1993a), S. 225-226 (wieder abegedruckt in Millikan (1993b), S. 186).

24 Siehe insbesondere Levine (1993) / deutsch in Pauen \& Stephan (2002), S. 91-102. 
Erklärungslücke lediglich ein epistemisches Problem ist - wir wissen nicht, wie wir den Erlebnischarakter von Bewusstseinszuständen mit etwas Physikalischem identifizieren können im Sinne der These der Identität der Vorkommnisse - oder ob diese Erklärungslücke eine ontologische Implikation hat und somit einen metaphysischen Dualismus nach sich zieht: Vorkommnisse von Bewusstseinszuständen sind aufgrund ihres Erlebnischarakters nicht mit Vorkommnissen [165] physikalischer Zustände identisch. ${ }^{25}$ Im letzteren Falle stellt sich das Problem der mentalen Verursachung in einer solchen Weise, dass eine epiphänomenalistische Konsequenz unausweichlich ist - es sei denn, man kann plausibel machen, entweder das Prinzip der Vollständigkeit physikalischer Ursachen (3) oder das Prinzip der Abwesenheit systematischer Überdetermination (4) aufzugeben.

Das Fazit dieses Artikels ist dieses: Das Problem des Regelfolgens ist ein starkes Argument für eine soziale, pragmatische Theorie begrifflichen Inhalts. Wenn begrifflicher Inhalt aber durch soziale, normative Praktiken bestimmt wird, dann stellt sich auch in Bezug auf den begrifflichen Inhalt intentionaler Zustände das Problem einer Erklärungslücke. Es ist zumindest nicht offensichtlich, wie für intentionale Zustände aufgrund des Holismus und des sozialen Externalismus eine These der Identität der Vorkommnisse mit Arrangements von Vorkommnissen physikalischer Zustände (Gehirnzuständen) gelten kann. Kurz, wie eine Erklärungslücke vom Nicht-Qualiamäßigen zu den Qualia besteht, so besteht eine Erklärungslücke vom Nicht-Sozial-Normativen zum Sozial-Normativen. Wiederum ist es fraglich, ob diese Erklàrungslücke lediglich ein epistemisches Problem anzeigt - wir wissen nicht, wie wir durch begrifflichen Inhalt charakterisierte intentionale Zuständen mit etwas Physikalischem identifizieren können im Sinne der These der Identität der Vorkommnisse oder ob diese Erklärungslücke eine ontologische Implikation hat und somit einen metaphysischen Dualismus nach sich zieht. Und wiederum gilt: Im letzteren Falle scheint eine epiphänomenalistische Konsequenz unausweichlich. Diese Konsequenz scheint im Falle von intentionalen Zuständen noch viel weniger etwas zu sein, mit dem wir zur Not leben könnten, als im Falle des Erlebnischarakters von Bewusstseinszuständen - gegeben, dass die sozialfunktionale Definition intentionaler Zustände unser Verständnis von uns selbst als handelnder Subjekte zu erfassen versucht.

Für eine soziale, pragmatische Theorie begrifflichen Inhalts ist es entscheidend, einen Ausweg aus diesem Problem der Erklärungslücke zu finden: Nur dann, wenn man zeigen kann, wie intentionale Zustände, verstanden im Sinne einer sozialen, pragmatischen Theorie begrifflichen Inhalts, Auswirkungen auf unser Verhalten haben, ist der Einwand entkräftet, eine solche Theorie könne die Realität intentionaler Zustände nicht aufweisen und laufe auf einen anti-realistischen Interpretationismus, einen Epiphänomenalismus oder einen eliminativen Materialismus hinaus. Gelingt es nicht, dieses zu zeigen, liegt ein hinreichender Grund dafür vor, die Argumente für eine solche Theorie begrifflichen Inhalts in Frage zu stellen und auf die Karte einer Naturalisierung der Semantik zu setzen.

\section{Literaturverzeichnis}

[166] Antony, Louise M. \& Levine, Joseph (1997): "Reduction with autonomy". In: J. E. Tomberlin (ed.): Philosophical Perspectives 11: Mind, causation, and world. Oxford: Blackwell. S. 83-105.

Averill, Edward \& Keating, B. F. (1981): “Does interactionism violate a law of classical physics?" Mind 90, S. 102-107. 
Bennett, Karen (2003): "Why the exclusion problem seems intractable, and how, just maybe, to tract it". Noûs 37, S. 471-497.

Brandom, Robert B. (1994): Making it explicit. Reasoning, representing, and discursive commitment. Cambridge (Massachusetts): Harvard University Press.

Brandom, Robert B. (2000): Articulating reasons. An introduction to inferentialism. Cambridge (Massachusetts): Harvard University Press.

Brandom, Robert B. (2000): Expressive Vernunft. Begründung, Repräsentation und diskursive Festlegung. Übersetzt von Eva Gilmer und Hermann Vetter. Frankfurt (Main): Suhrkamp.

Brandom, Robert B. (2001): Begründen und Begreifen. Eine Einführung in den Inferentialismus. Übersetzt von Eva Gilmer. Frankfurt (Main): Suhrkamp.

Chalmers, David J. (1996): The conscious mind. In search of a fundamental theory. New York: Oxford University Press.

Child, William (1994): Causality, interpretation and the mind. Oxford: Oxford University Press.

Davidson, Donald (1970): "Mental events". In: L. Foster \& J. W. Swanson (eds.): Experience and theory. Amherst: University of Massachusetts Press. S. 79-101. Wieder abgedruckt in Donald Davidson (1980): Essays on actions and events. Oxford: Oxford University Press. Essay 11.

Davidson, Donald (1984): Inquiries into truth and interpretation. Oxford: Oxford University Press.

Davidson, Donald (1985): Handlung und Ereignis. Übersetzt von Joachim Schulte. Frankfurt (Main): Suhrkamp.

Davidson, Donald (1986): Wahrheit und Interpretation. Übersetzt von Joachim Schulte. Frankfurt (Main): Suhrkamp.

Davidson, Donald (1993): “Thinking causes”. In: J. Heil \& A. Mele (eds.): Mental causation. Oxford: Oxford University Press. S. 3-17.

[167] Dennett, Daniel C. (1987): The intentional stance. Cambridge (Massachusetts): MIT Press.

Dennett, Daniel C. (1991): "Real patterns". Journal of Philosophy 88, S. 27-51.

Dretske, Fred I. (1989): "Reasons and causes". In: J. E. Tomberlin (ed.): Philosophical Perspectives 3: Philosophy of mind and action theory. Oxford: Blackwell. S. 1-15.

Esfeld, Michael (2000): "Is quantum indeterminism relevant to free will?" Philosophia Naturalis 37, S. $177-187$.

Esfeld, Michael (2002): Holismus in der Philosophie des Geistes und in der Philosophie der Physik. Frankfurt (Main): Suhrkamp.

Esfeld, Michael (2003): "L'argument sémantique pour la dépendance corporelle de la pensée". Studia Philosophica 62, S. 119-131.

Haugeland, John (1998): Having thought. Essays in the metaphysics of mind. Cambridge (Massachusetts): Harvard University Press.

Kim, Jaegwon (1993): “Can supervenience and 'non-strict' laws save anomalous monism?” In: J. Heil \& A. Mele (eds.): Mental causation. Oxford: Oxford University Press. S. 19-26.

Kim, Jaegwon (1998): Mind in a physical world. An essay on the mind-body problem and mental causation. Cambridge (Massachusetts): MIT Press.

Kripke, Saul A. (1982): Wittgenstein on rules and private language. Oxford: Blackwell.

Kripke, Saul A. (1987): Wittgenstein über Regeln und Privatsprache. Übersetzt von Helmut Pape. Frankfurt (Main): Suhrkamp.

Levine, Joseph (1993): “On leaving out what it's like”. In: M. Davies \& G. W. Humphreys (eds.): Consciousness. Psychological and philosophical essays. Oxford: Blackwell. S. 121-136.

Loewer, Barry (2001): "Review of Jaegwon Kim, Mind in a physical World. An essay on the mind-body problem and mental causation, Cambridge (Massachusetts): MIT Press 1998". Journal of Philosophy 98, S. $315-324$.

McDowell, John (1994): Mind and world. Cambridge (Massachusetts): Harvard University Press.

McDowell, John (1998): Geist und Welt. Übersetzt von Thomas Blume, Holm Bräuer, Gregory Klass. Paderborn: Schöningh. 
[168] McLaughlin, Brian P. (1993): “On Davidson's reponse to the charge of epiphenomenalism”. In: J. Heil \& A. Mele (eds.): Mental causation. Oxford: Oxford University Press. S. 27-40.

Millikan, Ruth Garrett (1993a): "Explanation in biopsychology”. In: J. Heil \& A. Mele (eds.): Mental causation. Oxford: Oxford University Press. S. 211-232.

Millikan, Ruth Garrett (1993b): White queen psychology and other essays for Alice. Cambridge (Massachusetts): MIT Press.

Pauen, Michael \& Stephan, Achim (Hgg.) (2002): Phänomenales Bewußtsein - Rückkehr zur Identitätstheorie? Paderborn: Mentis.

Pettit, Philip (1993): The common mind. An essay on psychology, society, and politics. Oxford: Oxford University Press.

Poland, Jeffrey (1994): Physicalism. The philosophical foundations. Oxford: Oxford University Press.

Putnam, Hilary (1999): The threefold cord: mind, body, and world. New York: Columbia University Press.

Sellars, Wilfrid (1956): "Empiricism and the philosophy of mind". In: H. Feigl \& M. Scriven (eds.): The foundations of science and the concepts of psychology and psychoanalysis. Minnesota Studies in the philosophy of science. Volume 1. Minneapolis: University of Minnesota Press. S. 253-329.

Sellars, Wilfrid (1997): Empiricism and the philosophy of mind. With an introduction by Richard Rorty and a study guide by Robert Brandom. Cambridge (Massachusetts): Harvard University Press.

Sellars, Wilfrid (1999): Der Empirismus und die Philosophie des Geistes. Übersetzt von Thomas Blume. Paderborn: Mentis.

Shoemaker, Sydney (1981): "Some varieties of functionalism". Philosophical Topics 12, S. 83-118. Wieder abgedruckt in Sydney Shoemaker (1984): Identity, cause, and mind. Philosophical essays. Cambridge: Cambridge University Press. S. 261-286.

Sosa, Ernest (1993): “Davidson's thinking causes". In: J. Heil \& A. Mele (eds.): Mental causation. Oxford: Oxford University Press. S. 41-50.

Wittgenstein, Ludwig (1953): Philosophische Untersuchungen. Hgg. G.E.M. Anscombe, G.H. von Wright, Rush Rhees. In: Ludwig Wittgenstein. Werkausgabe in 8 Bänden. Band 1. Frankfurt (Main): Suhrkamp 1984.

Yablo, Stephen (1992): "Mental causation”. Philosophical Review 101, S. 245-280. 